\title{
Performance Evaluation of Location Management in UMTS
}

\author{
Shun-Ren Yang* and Yi-Bing Lin
}

\begin{abstract}
Universal Mobile Telecommunications System (UMTS) utilizes a three-level location management strategy to reduce the net costs of location update and paging in the packet-switched service domain. Within a communication session, a mobile station (MS) is tracked at the cell level during packet transmission. In the idle period of an ongoing session, the MS is tracked at the UTRAN registration area (URA) level to avoid frequent cell updates while still keeping the radio connection. If the MS is not in any communication session, the MS is tracked at the routing area (RA) level. The inactivity counter mechanism was proposed in 3GPP 25.331 to determine when to switch between the three location tracking modes. In this mechanism, two inactivity counters are used to count the numbers of cell updates and URA updates in an idle period between two packet transmissions. If the number of cell updates reaches a threshold $K_{1}$, the MS is switched from the cell tracking to the URA tracking. After that, if the number of URA updates reaches a threshold $K_{2}$, the MS is tracked at the RA level. This paper proposes analytical and simulation models to investigate the performance of the inactivity counter mechanism. Our study provides guidelines for $K_{1}$ and $K_{2}$ selection to achieve lower net costs of location update and paging.
\end{abstract}

Keywords: UMTS, mobile network, mobility management, wireless data.

${ }^{*}$ Corresponding Author: Shun-Ren Yang, Dept. Comp. Sci. \& Info. Engr., National Chiao Tung University, Hsinchu, Taiwan, R.O.C.; Fax:+886-3.5724176; Email: sjyoun@csie.nctu.edu.tw 


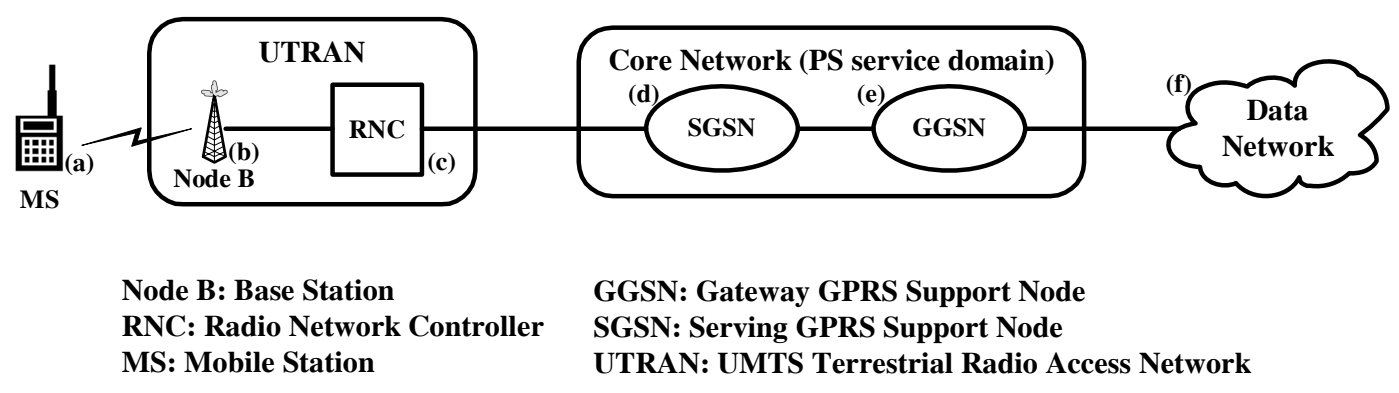

Figure 1: A simplified network architecture for the UMTS PS domain

\section{Introduction}

Existing second generation (2G) mobile communications systems (such as GSM) are designed for voice services, which only have limited capabilities for offering data services. On the other hand, the third generation (3G) systems such as Universal Mobile Telecommunications System (UMTS) [10] support mobile multimedia applications with high data transmission rates. As shown in Figure 1, the UMTS infrastructure includes the Core Network $(\mathrm{CN})$ and the UMTS Terrestrial Radio Access Network (UTRAN). The CN is responsible for switching/routing calls and data connections to the external networks, while the UTRAN handles all radio-related functionalities. The CN consists of two service domains: the Circuit-Switched (CS) service domain and the PacketSwitched (PS) service domain. The CS domain provides the access to the PSTN/ISDN, while the PS domain provides the access to the IP-based networks. In the remainder of this paper, we will focus on the UMTS packet switching mechanism. In the PS domain of the $\mathrm{CN}$, the packet data services of a Mobile Station (MS; see Figure 1(a)) are provided by the Serving GPRS Support Node (SGSN; see Figure 1(d)) and the Gateway GPRS Support Node (GGSN; see Figure 1(e)). The SGSN connects the MS to the external data network through the GGSN. The UTRAN consists of Node Bs (the 3G term for base stations; see Figure 1(b)) and Radio Network Controllers (RNCs; see Figure 1(c)) connected by an ATM network. The connection between the UTRAN and the CN is achieved via the ATM links between the RNCs and the SGSNs. The MS communicates with Node Bs through the radio interface based on the WCDMA (Wideband CDMA) technology.

The cells (i.e., radio coverages of Node Bs) in a UMTS service area are partitioned into several groups. To deliver services to an MS, the cells in the group covering the MS will page the MS to establish the radio connection. The location change of an MS is detected as follows. The 


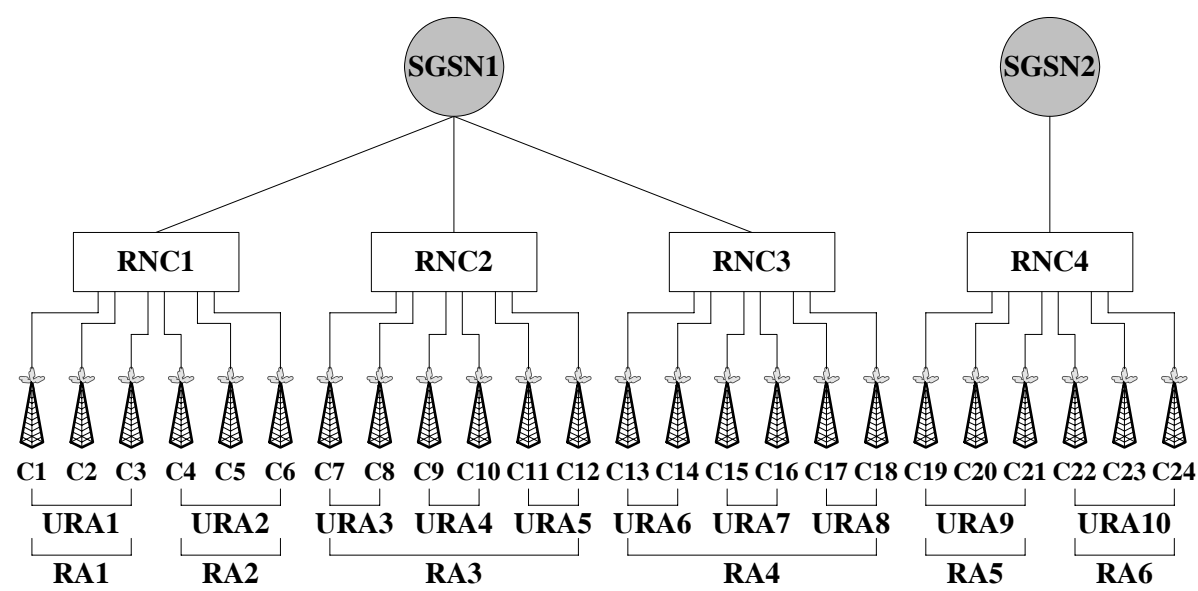

Figure 2: Cells, URAs and RAs

cells periodically broadcast their cell identities. The MS listens to the broadcast cell identity, and compares it with the cell identity stored in the MS's buffer. If the comparison indicates that the location has been changed, then the MS sends the location update message to the network. In the UMTS PS domain, the cells are grouped into Routing Areas (RAs). The RA of an MS is tracked by the SGSN. The cells in an RA are further grouped into UTRAN Registration Areas (URAs). The URA and the cell of an MS are tracked by the UTRAN. Figure 2 illustrates an example layout of cells, URAs and RAs.

In UMTS, the mobility management activities for an MS are characterized by two finite state machines: Mobility Management (MM) state machine and Radio Resource Control (RRC) state machine. The Packet MM (PMM) state machine for the UMTS PS domain is exercised between the SGSN and the MS for CN-level tracking, while the RRC state machine is executed between the UTRAN and the MS for UTRAN-level tracking. Incomplete state diagrams for these two machines are illustrated in Figure 3. Specifically, the figure only considers the states after the MS has attached to the PS domain. The PMM state diagram in the MS is slightly different from that in the SGSN. To simplify the presentation, we only show the common portions of the MS and the SGSN state transitions that are used in this paper. The state diagrams in Figure 3 are described as follows. After an MS is attached to the PS service domain, the PMM state machine will be in one of the two states: PMM-IDLE and PMM-CONNECTED. In the RRC state machine, there are three states: RRC Idle Mode, RRC Cell Connected Mode and RRC URA Connected Mode. We will briefly elaborate on the PMM and RRC state transitions. The readers are referred to [2] 
and [1] for complete descriptions of the PMM and RRC state machines.

When there is no data transmission between the MS and the core network, the MS is in the PMMIDLE state and RRC Idle Mode. In this case, UTRAN has no information about the idle MS, and the MS is tracked by the SGSN at the RA level.

When a PS signaling connection is established between the MS and the SGSN (possibly in response to a page from the SGSN), the MS enters the PMM-CONNECTED state (see T1 in Figure 3(a)). Since the establishment of the PS signaling connection triggers the establishment of the RRC connection between the MS and its serving RNC, the RRC state of the MS is switched to RRC Cell Connected Mode (see T1 in Figure 3(b)). In this case, the SGSN tracks the MS with accuracy of the serving RNC, and the serving RNC is responsible for tracking the cell where the MS resides. Packets can only be delivered in this state.

In the PMM-CONNECTED/RRC Cell Connected Mode, if the MS has not transmitted/received packets for a period, the RRC state of the MS is switched to RRC URA Connected Mode (see T2 in Figure 3(b)). In this case, the RRC connection is still maintained while the URA of the MS is tracked by the serving RNC. In this transition, the PMM state of the MS remains unchanged; i.e., the state is PMM-CONNECTED.

In the PMM-CONNECTED/RRC URA Connected Mode, if the MS transmits/receives a packet, the RRC state is moved back to RRC Cell Connected Mode (see T3 in Figure 3(b)). On the other hand, if the PS signaling connection and the RRC connection are released (e.g., a communication session is completed), or if no packet is transmitted for a long time, the RRC state is first switched to RRC Cell Connected Mode and then to RRC Idle Mode (see T3 and T4 in Figure 3(b)). In this case, the PMM state is also changed to PMM-IDLE (see T4 in Figure 3(a)).

The above three-level location management strategy is designed to reduce the net costs of location update and paging. Within a communication session (i.e., the MS is in the RRC Cell Connected Mode and the PMM-CONNECTED state), the MS expects to continuously transmit/receive packets. In this case, the MS performs location update whenever it moves to a new cell. With cell update, no cell is paged if there are dedicated channels allocated to the MS. If the MS has no dedicated channel, the currently visited cell is requested to page the MS for packet delivery. Without loss of generality, we assume one cell paging cost in this paper. On the other hand, during an ongoing session, there may exist some idle periods where no packets are delivered. To avoid frequent cell 


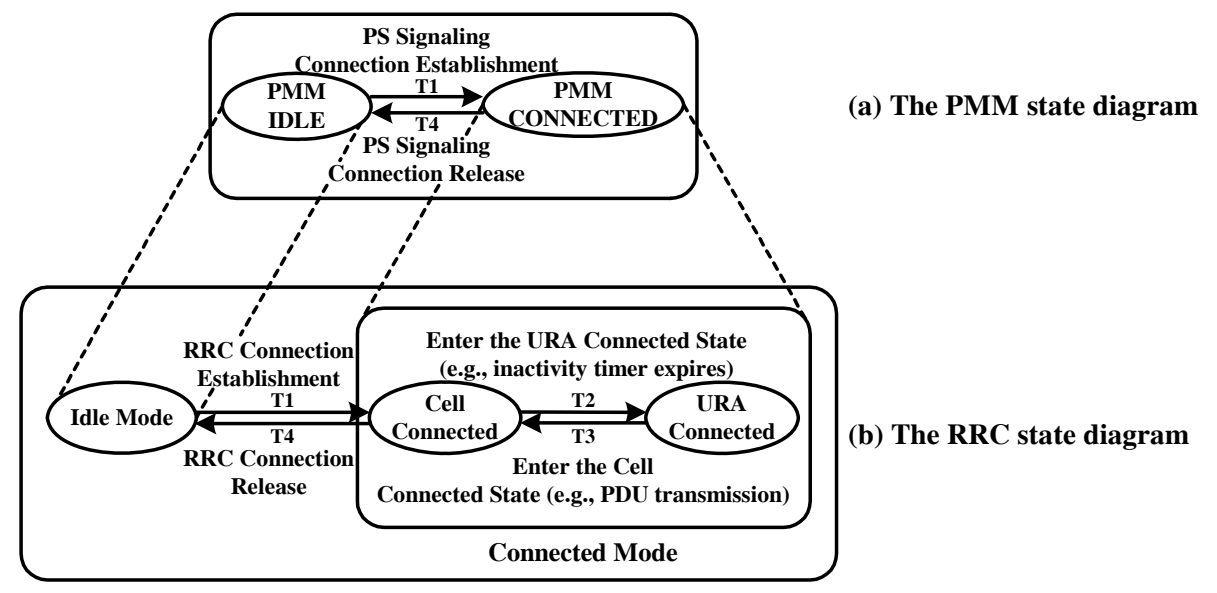

Figure 3: State diagrams for UMTS mobility management: (a) an incomplete PMM state diagram for PS domain; (b) a simplified RRC state diagram

Table 1: The UMTS location update costs

\begin{tabular}{|l|c|c|c|}
\hline location update modes & cell update & URA update & RA update \\
\hline location update cost & high & medium & low \\
\hline paging cost & low & medium & high \\
\hline
\end{tabular}

updates while still keeping the RRC connection in this situation, the MS is switched to RRC URA Connected Mode to perform URA update for every URA crossing. When the communication session between the MS and the SGSN is completed, the MS will not transmit/receive any packet for a long period. In this case, the RRC connection should be released for efficient wireless bandwidth utilization. In addition, the MS should be tracked at the RA level because cell level or URA level tracking is too expensive in terms of signaling overhead and power consumption. Therefore, the RRC and PMM states are switched to RRC Idle Mode and PMM-IDLE state, respectively, and the MS is tracked at the RA level. Based on the above description, the costs of the three location update modes are summarized in Table 1.

In the PMM and RRC state machines, the mechanism that triggers transitions $\mathbf{T} 2$ and $\mathbf{T 4}$ has significant impacts on the signaling traffic of the UMTS system. This mechanism can be implemented by two approaches. The first approach makes use of two inactivity timers $X_{1}$ and $X_{2}$. At the end of a packet transmission, timer $X_{1}$ is set to a predefined threshold value and is decremented as 
time elapses. Transition T2 occurs if the MS does not transmit/receive any packet before timer $X_{1}$ expires. When timer $X_{1}$ expires, the second timer $X_{2}$ is set to a predefined threshold value and is decremented. Timer $X_{2}$ is used to determine the time when transition T4 occurs. In the second approach, two inactivity counters $Y_{1}$ and $Y_{2}$ are employed. Counter $Y_{1}$ counts the number of cell updates in the idle period between two packet transmissions. If the number of cell updates reaches a threshold $K_{1}$, then the MS is switched to perform URA updates through transition T2. After T2 has occurred, counter $Y_{2}$ is used to count the number of URA updates in the observed idle period. If the number of URA updates reaches a threshold $K_{2}$, then the MS is switched to perform RA updates (i.e., transition $\mathbf{T} 4$ occurs).

As pointed out in our previous work [12], the timer approach may have synchronization problem. That is, the peer state machines in the MS and the UTRAN or the SGSN may stay in different states at the same time due to the errors of the clock rates. Besides, the counter approach may significantly outperform the timer approach for the following reason. The timer approach uses two timers of fix-length thresholds. When the mobility rate and/or packet transmission patterns change, the fixed thresholds of the timers do not adapt to the changes. On the other hand, the thresholds $K_{1}$ and $K_{2}$ of the counter approach always capture the $K_{1}$-th cell update and $K_{2}$-th URA update of an MS no matter how the mobility rate and packet transmission patterns change. Therefore, this paper will not elaborate on the inactivity timer approach and only focus on the inactivity counter mechanism. In the following sections, we propose analytical and simulation models to investigate the performance of the inactivity counter mechanism. Specifically, given any mobility and traffic patterns, we determine the net costs of location update and paging under various $K_{1}$ and $K_{2}$ threshold values. Our study provides guidelines for $K_{1}$ and $K_{2}$ selection that results in lower net costs.

\section{Analytical Model for Inactivity Counter Mechanism}

This section proposes an analytical model to study the UMTS inactivity counter mechanism. We first describe a two-dimensional random walk model for user movement based on a hexagonal cellular configuration. Then we show how to use this model to investigate the performance of the UMTS inactivity counter mechanism. For specific thresholds $K_{1}$ and $K_{2}$, we derive the expected number of location updates (including cell updates, URA updates and RA updates) performed in 


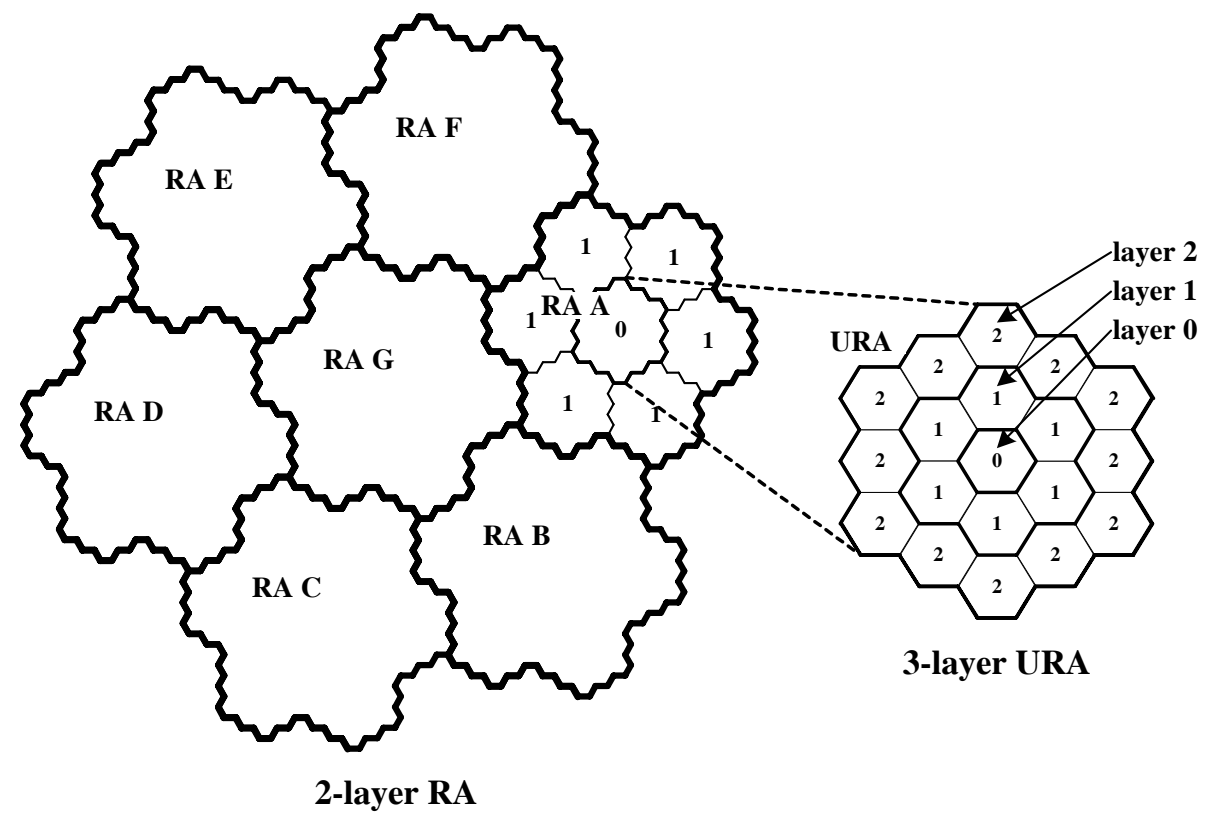

Figure 4: Cell/URA/RA layout in a UMTS Network

the idle period between two packet transmissions, and the expected number of cells need to be paged for packet delivery.

\subsection{The Random Walk Model}

For demonstration purposes, we consider the hexagonal cell layout in Figure 4. In this configuration, the cells are clustered into several UTRAN Registration Areas (URAs), and the URAs are in turn clustered into several Routing Areas (RAs). An $n$-layer URA covers $S(n)=3 n^{2}-3 n+1$ cells. Figure 4 illustrates a 3-layer URA. The cell at the center of the URA is referred to as the layer 0 cell. The cells surrounding layer $x-1$ cells are referred to as the layer $x$ cells. There are $6 x$ cells in layer $x$ except for layer 0 that contains exactly one cell. An $n$-layer URA consists of cells from layer 0 to layer $n$-1. The structure of an $n$-layer RA is similar to that of an $n$-layer URA except that the basic elements are URAs instead of cells. Therefore, an $n$-layer RA covers $S(n)$ URAs. Figure 4 illustrates seven 2-layer RAs (A, B, C, D, E, F and G). Each of them consists of seven 3-layer URAs. Based on this RA/URA/cell structure, we derive the number of cells and URAs that are visited before a user moves out of an RA.

In [3], we proposed a cell type classification algorithm based on the random walk model. The work 


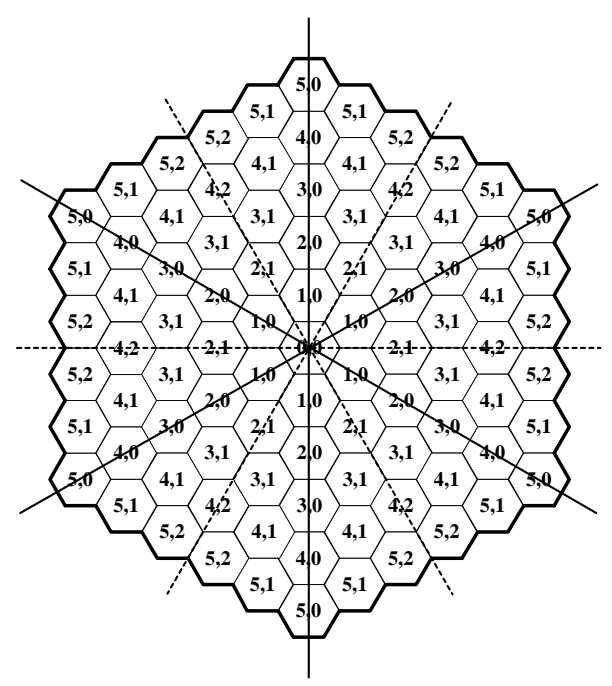

Figure 5: Type classification for a 6-layer UTRAN registration area

showed that an $n$-layer hexagonal cellular network can be modeled by a state transition diagram with $n(n+1) / 2$ states. Based on our model, Tseng et al. [16] further reduced the number of states to $A(n)$, where for $n>1$

$$
A(n)= \begin{cases}\frac{(n+1)(n+3)}{4}, & n \text { is odd } \\ \frac{n(n+4)}{4}, & n \text { is even }\end{cases}
$$

According to the type classification algorithm in $[3,16]$, we classify the cells in a URA into several cell types. For $x \geq 0$ and $y \geq 0$, a cell type is of the form $\langle x, y\rangle$, where $x$ represents that the cell is in layer $x$, and $y$ represents the $y$ th type in layer $x$. Cells of the same type are indistinguishable in terms of movement pattern because they are at the symmetrical positions (with respect to the solid and dashed lines in Figure 5) on the hexagonal URA. Based on the derivation in [16], Figure 5 labels the types of cells for a 6-layer URA. In this example, the cell in layer 0 is of the type $\langle 0,0\rangle$. The six cells in layer 1 are grouped together and assigned to the same type $\langle 1,0\rangle$. A layer 2 cell may have three or two neighbors in layer 3 and is assigned to types $\langle 2,0\rangle$ and $\langle 2,1\rangle$, respectively.

Based on the above cell labeling, we compute the number of movements an MS will take to cross the boundary of an $n$-layer URA. A state of this random walk is of the form $(x, y)$. For $0 \leq x<n$ and $0 \leq y \leq\lfloor x / 2\rfloor$, the state $(x, y)$ is transient, which represents that the MS is in one of the cells of type $\langle x, y\rangle$. For $x=n$ and $0 \leq y \leq\lfloor(n-1) / 2\rfloor$, the state $(n, y)$ is absorbing, which represents that the MS crosses the boundary of the URA from a cell of type $\langle n-1, y\rangle$. Details of the random 
walk and derivation of its steady state probabilities are given in [3]. The results are summarized as follows. Let $p_{(x, y),\left(x^{\prime}, y^{\prime}\right)}$ be the one-step transition probability from state $(x, y)$ to state $\left(x^{\prime}, y^{\prime}\right)$, i.e., the probability that the MS moves from a $\langle x, y\rangle$ cell to a $\left\langle x^{\prime}, y^{\prime}\right\rangle$ cell in one step. The transition probability matrix $P=\left(p_{(x, y),\left(x^{\prime}, y^{\prime}\right)}\right)$ of the random walk is given below.

$$
P=\left(\begin{array}{ccccccccc}
0 & 1 & 0 & 0 & 0 & \cdots & 0 & 0 & 0 \\
1 / 6 & 1 / 3 & 1 / 6 & 1 / 6 & 0 & \cdots & 0 & 0 & 0 \\
0 & 1 / 6 & 0 & 1 / 3 & 1 / 6 & \cdots & 0 & 0 & 0 \\
0 & 1 / 3 & 1 / 3 & 0 & 0 & \cdots & 0 & 0 & 0 \\
0 & 0 & 1 / 6 & 0 & 0 & \cdots & 0 & 0 & 0 \\
\vdots & \vdots & \vdots & \vdots & \vdots & \ddots & \vdots & \vdots & \vdots \\
0 & 0 & 0 & 0 & 0 & \cdots & 1 & 0 & 0 \\
0 & 0 & 0 & 0 & 0 & \cdots & 0 & 1 & 0 \\
0 & 0 & 0 & 0 & 0 & \cdots & 0 & 0 & 1
\end{array}\right)_{A(n) \times A(n)}
$$

As an example, the element $p_{12}=1 / 6$ in matrix $P$ represents that the MS moves from a $\langle 1,0\rangle$ cell to a $\langle 2,0\rangle$ cell in one step with probability $1 / 6$. Define $P^{(k)}$ as follows.

$$
P^{(k)}= \begin{cases}P, & \text { for } k=1 \\ P^{(k-1)} P, & \text { for } k>1\end{cases}
$$

An element $p_{(x, y),\left(x^{\prime}, y^{\prime}\right)}^{(k)}$ in $P^{(k)}$ is the probability that the random walk moves from state $(x, y)$ to state $\left(x^{\prime}, y^{\prime}\right)$ with $k$ steps (it is possible that state $\left(x^{\prime}, y^{\prime}\right)$ is re-visited several times during these $k$ steps). Let $p_{k,(x, y),\left(x^{\prime}, y^{\prime}\right)}$ be the probability that an MS initially stays at state $(x, y)$, and takes its first entrance into state $\left(x^{\prime}, y^{\prime}\right)$ at the $k$ th step. Then $p_{k,(x, y),(n, j)}$ is expressed as

$$
p_{k,(x, y),(n, j)}= \begin{cases}p_{(x, y),(n, j)}, & \text { for } k=1 \\ p_{(x, y),(n, j)}^{(k)}-p_{(x, y),(n, j)}^{(k-1)}, & \text { for } k>1\end{cases}
$$

which can be solved by using the transition probability matrices (2) and (3). Equation (4) gives the probability that an MS will move out of a URA at the $k$ th cell crossing. Note that the number of URA movements before the user leaves an RA can also be derived using the same approach where the cells are replaced by the URAs.

\subsection{Location Update and Paging Costs}

Figure 6 shows the timing diagram of the location update activities for an MS in an idle period between two packet transmissions. Suppose that the previous packet transmission of the MS ends at time $t_{0}$ and the next packet transmission begins at time $t_{1}$. Let $t_{p}=t_{1}-t_{0}$. Consider the 


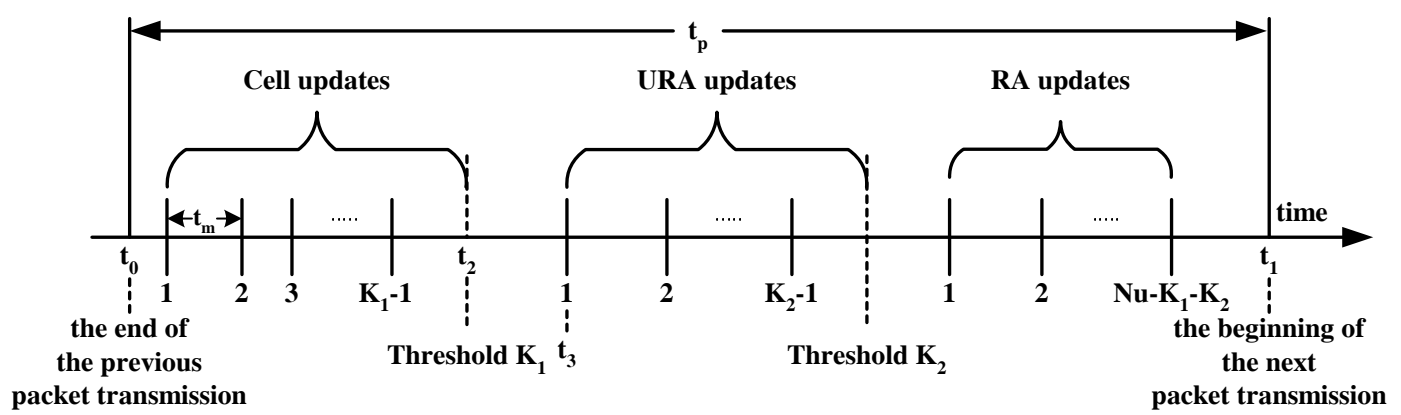

Figure 6: Timing diagram for cell, URA and RA updates

execution of the inactivity counter mechanism with specific thresholds $K_{1}$ and $K_{2}$. For the first $K_{1}$ cell crossings during period $t_{p}$, the MS performs a cell update for each of the cell crossings. After the $K_{1}$-th cell update, the MS will not perform any cell update and starts performing the URA updates. After the $K_{2}$-th URA update, the MS will not perform any URA update and starts performing the RA updates. Assume that the $K_{1}$-th cell update occurs at time $t_{2}$ and the first URA update occurs at time $t_{3}$. Based on the equal routing probability assumption of the random walk, at time $t_{2}$, the MS will stay at any cell of the visited URA with equal probability. On the other hand, after any URA update, the MS will only stay at one of the boundary cells of the visited URA. Thus, to derive the number of URA updates in $t_{p}$, we need to consider the following two cases.

Case I. Starting from an arbitrary cell, we compute the number of cell crossings before the MS moves out of the URA (i.e., the number of cell crossings in $\left(t_{2}, t_{3}\right]$ in Figure 6). As we mentioned before, at time $t_{2}$ the MS can be in any cell of the URA with the same probability. Therefore at $t_{2}$, the MS is at the $\langle 0,0\rangle$ cell with probability $1 / S(n)$, at a cell of type $\langle x, 0\rangle$ $(1 \leq x<n)$ with probability $6 / S(n)$, at a cell of type $\langle 2 x, x\rangle(1 \leq x \leq\lfloor(n-1) / 2\rfloor)$ with probability $6 / S(n)$, and at a cell of type $\langle x, y\rangle(3 \leq x<n, 1 \leq y \leq\lfloor(x-1) / 2\rfloor)$ with probability $12 / S(n)$, where

$$
S(n)=3 n^{2}-3 n+1
$$

is the number of cells covered by an $n$-layer URA. Consider a 6-layer URA that covers $S(6)=91$ cells (see Figure 5). Since there are six cells of type $\langle 2,1\rangle$ and twelve cells of type $\langle 3,1\rangle$ in the URA, the MS is in a cell of type $\langle 2,1\rangle$ with probability $6 / 91$ and in a cell of type $\langle 3,1\rangle$ with probability $12 / 91$. Let $\theta_{1}(n, k)$ be the probability that after $t_{2}$, the MS 
will leave the $n$-layer URA at the $k$ th cell movement. Then

$$
\begin{aligned}
\theta_{1}(n, k) & =\left[\frac{1}{S(n)}\right]\left[\sum_{j=0}^{\lfloor(n-1) / 2\rfloor} p_{k,(0,0),(n, j)}\right]+\left[\frac{12}{S(n)}\right]\left[\sum_{x=3}^{n-1} \sum_{y=1}^{\lfloor(x-1) / 2\rfloor} \sum_{j=0}^{\lfloor(n-1) / 2\rfloor} p_{k,(x, y),(n, j)}\right] \\
& +\left[\frac{6}{S(n)}\right]\left[\sum_{x=1}^{n-1} \sum_{j=0}^{\lfloor(n-1) / 2\rfloor} p_{k,(x, 0),(n, j)}+\sum_{x=1}^{\lfloor(n-1) / 2\rfloor} \sum_{j=0}^{\lfloor(n-1) / 2\rfloor} p_{k,(2 x, x),(n, j)}\right]
\end{aligned}
$$

Case II. Starting from a boundary cell of an URA at time $t_{3}$, we compute the number of cell crossings before the MS moves out of the URA. In [13], we showed that after entering the URA, the MS is in a boundary cell with probability proportional to the number of boundary edges for that boundary cell. Under the condition that an MS is moving into a boundary cell, the MS enters a type $\langle n-1,0\rangle$ cell with probability $3 \cdot 6 / B(n)$, enters a type $\langle n-1,(n-1) / 2\rangle$ cell (when $n$ is odd) with probability $2 \cdot 6 / B(n)$, and enters a type $\langle n-1, y\rangle$ cell $(1 \leq y \leq$ $\lfloor n / 2\rfloor-1$ ) with probability $2 \cdot 12 / B(n)$, where $B(n)=6[3+2(n-2)]$ is the number of boundary edges in an $n$-layer URA. In Figure $5, B(n)=66$ for a 6-layer URA. In this example, there are three boundary edges for each of the six $\langle 5,0\rangle$ cells, and the MS enters a boundary cell of type $\langle 5,0\rangle$ with probability $18 / 66$. Similarly, there are two boundary edges for each of the twelve $\langle 5,2\rangle$ cells, and the MS enters a boundary cell of type $\langle 5,2\rangle$ with probability $24 / 66$. Let $\theta_{2}(n, k)$ be the probability that after an MS enters an $n$-layer URA, it moves out of the URA at the $k$ th cell movement. If $n$ is odd, then

$$
\begin{aligned}
\theta_{2}(n, k) & =\left[\frac{3 \cdot 6}{B(n)}\right]\left[\sum_{j=0}^{\lfloor(n-1) / 2\rfloor} p_{k,(n-1,0),(n, j)}\right]+\left[\frac{2 \cdot 6}{B(n)}\right]\left[\sum_{j=0}^{\lfloor(n-1) / 2\rfloor} p_{k,(n-1,(n-1) / 2),(n, j)}\right] \\
& +\left[\frac{2 \cdot 12}{B(n)}\right]\left[\sum_{y=1}^{\lfloor n / 2\rfloor-1} \sum_{j=0}^{\lfloor(n-1) / 2\rfloor} p_{k,(n-1, y),(n, j)}\right]
\end{aligned}
$$

If $n$ is even, then

$$
\theta_{2}(n, k)=\left[\frac{3 \cdot 6}{B(n)}\right]\left[\sum_{j=0}^{\lfloor(n-1) / 2\rfloor} p_{k,(n-1,0),(n, j)}\right]+\left[\frac{2 \cdot 12}{B(n)}\right]\left[\sum_{y=1}^{\lfloor n / 2\rfloor-1} \sum_{j=0}^{\lfloor(n-1) / 2\rfloor} p_{k,(n-1, y),(n, j)}\right]
$$

Suppose that an MS is in an arbitrary cell of an $n$-layer URA. Let $P\left(n, N_{m}, k\right)$ be the probability that after $N_{m}$ cell movements, the MS crosses $k$ URA boundaries. Similarly, consider an MS initially residing at a boundary cell of an $n$-layer URA. Let $P^{*}\left(n, N_{m}^{*}, k^{*}\right)$ be the probability that 
after $N_{m}^{*}$ cell movements, the MS crosses $k^{*}$ URA boundaries. From (6), (7) and (8), we have

$$
P\left(n, N_{m}, k\right)= \begin{cases}1, \sum_{j=N_{m}+1}^{\infty} \theta_{1}(n, j), & \text { for } k=N_{m}=0 \\ \sum_{j=1}^{N_{m}} \theta_{1}(n, j) P^{*}\left(n, N_{m}-j, k-1\right), & \text { for } k \geq 0, N_{m}>0 \\ 0, & \text { for } N_{m}<k\end{cases}
$$

Four cases are considered in Equation (9).

Case I: $k=N_{m}=0$. If there is no cell movement during an idle period, there will be no URA boundary crossing during this period, and $P\left(n, N_{m}=0, k=0\right)=1$.

Case II: $k=0, N_{m}>0$. Assume that the MS crosses the first URA boundary at the $j$ th cell movement (with probability $\theta_{1}(n, j)$ ). For $N_{m}>0$, if $k=0$ (i.e., the MS does not cross any URA boundary during these $N_{m}$ cell movements), $j$ must be larger than $N_{m}$. Therefore in this case, $P\left(n, N_{m}, k\right)=\operatorname{Pr}\left[j>N_{m}\right]=\sum_{j=N_{m}+1}^{\infty} \theta_{1}(n, j)$.

Case III: $k \geq 1, N_{m} \geq k$. In this case, there are $k$ URA boundary crossings during the $N_{m}$ cell movements. If the MS crosses the first URA boundary at the $j$ th cell movement (with probability $\left.\theta_{1}(n, j)\right)$, then the MS must cross the remaining $k-1$ URA boundaries during the subsequent $N_{m}-j$ cell movements (with probability $P^{*}\left(n, N_{m}-j, k-1\right)$ ). Therefore, $P\left(n, N_{m}, k\right)=\sum_{j=1}^{N_{m}} \theta_{1}(n, j) P^{*}\left(n, N_{m}-j, k-1\right)$.

Case IV: $N_{m}<k$. During an idle period, it is impossible that the number of URA boundary crossings is larger than the number of cell movements. Thus, $P\left(n, N_{m}, k\right)=0$ in this case.

Similarly, we have

$$
P^{*}\left(n, N_{m}^{*}, k^{*}\right)= \begin{cases}1, & \text { for } k^{*}=N_{m}^{*}=0 \\ \sum_{j=N_{m}^{*}+1}^{\infty} \theta_{2}(n, j), & \text { for } k^{*}=0, N_{m}^{*}>0 \\ \sum_{j=1}^{N_{m}^{*}} \theta_{2}(n, j) P^{*}\left(n, N_{m}^{*}-j, k^{*}-1\right), & \text { for } k^{*} \geq 1, N_{m}^{*} \geq k^{*} \\ 0, & \text { for } N_{m}^{*}<k^{*}\end{cases}
$$

Equations (9) and (10) can be effectively computed by using the dynamic programming technique [14]. Note that equations (6)-(10) can also be used to derive the number of RA boundary crossings for an $n$-layer RA layout given that the number of URA movements is known. 
With (9) and (10), we derive the number of cell/URA/RA updates in the idle period between two packet transmissions as follows. Assume that $t_{p}=t_{1}-t_{0}$ in Figure 6 has a general distribution with the density function $f_{p}\left(t_{p}\right)$, the expected value $1 / \lambda_{p}$ and the Laplace Transform

$$
f_{p}^{*}(s)=\int_{t_{p}=0}^{\infty} e^{-s t_{p}} f_{p}\left(t_{p}\right) d t_{p}
$$

For the inactivity counter mechanism with specific thresholds $K_{1}$ and $K_{2}$, let $N_{u}$ be the number of location updates (including the cell updates, the URA updates and the RA updates) during period $t_{p}$. Based on the aforementioned random walk model, the distribution of $N_{u}$ can be derived as follows. Suppose that the cell residence time $t_{m}$ has an Erlang distribution with mean $1 / \lambda_{m}=$ $m / \lambda$, variance $V_{m}=m / \lambda^{2}$, and density function

$$
f_{m}\left(t_{m}\right)=\frac{\lambda e^{-\lambda t_{m}}\left(\lambda t_{m}\right)^{m-1}}{(m-1) !} \text { for } t_{m} \geq 0
$$

where $m=1,2,3, \ldots$ We select the Erlang distribution because this distribution can be easily extended into a hyper-Erlang distribution. The hyper-Erlang distribution has been proven as a good approximation to many other distributions as well as measured data $[7,8,11]$.

Since the cell crossings of an MS can be modeled as an equilibrium Erlang renewal process [15], the probability mass function of the number of cell crossings $N_{c}$ within $t_{p}$ is

$$
\begin{aligned}
\operatorname{Pr}\left[N_{c}=k\right]= & \int_{t_{p}=0}^{\infty}\left(\frac{e^{-\lambda t_{p}}}{m}\right)\left\{\sum_{j=k m}^{k m+m-1}\left[\frac{(k m+m-j)\left(\lambda t_{p}\right)^{j}}{j !}\right]\right. \\
& \left.-\sum_{j=k m-m}^{k m-1}\left[\frac{(j-k m+m)\left(\lambda t_{p}\right)^{j}}{j !}\right]\right\} f_{p}\left(t_{p}\right) d t_{p} \\
= & \left(\frac{1}{m}\right)\left\{\sum_{j=k m}^{k m+m-1}\left[\frac{(k m+m-j) \lambda^{j}}{j !}\right]\left[\int_{t_{p}=0}^{\infty} t_{p}^{j} f_{p}\left(t_{p}\right) e^{-\lambda t_{p}} d t_{p}\right]\right. \\
& -\sum_{j=k m-m}^{k m-1}\left[\frac{(j-k m+m) \lambda^{j}}{j !}\left[\int_{t_{p}=0}^{\infty} t_{p}^{j} f_{p}\left(t_{p}\right) e^{-\lambda t_{p}} d t_{p}\right]\right\} \\
= & \left(\frac{1}{m}\right)\left\{\left.\sum_{j=k m}^{k m+m-1}\left[\frac{(k m+m-j)(-\lambda)^{j}}{j !}\right]\left[\frac{d^{j} f_{p}^{*}(s)}{d s^{j}}\right]\right|_{s=\lambda}\right. \\
& \left.-\left.\sum_{j=k m-m}^{k m-1}\left[\frac{(j-k m+m)(-\lambda)^{j}}{j !}\right]\left[\frac{d^{j} f_{p}^{*}(s)}{d s^{j}}\right]\right|_{s=\lambda}\right\}
\end{aligned}
$$

for $k=1,2, \ldots$ For $k=0$, we have

$$
\operatorname{Pr}\left[N_{c}=0\right]=\int_{t_{p}=0}^{\infty}\left(\frac{e^{-\lambda t_{p}}}{m}\right) \sum_{j=0}^{m-1}\left[\frac{(m-j)\left(\lambda t_{p}\right)^{j}}{j !}\right] f_{p}\left(t_{p}\right) d t_{p}
$$




$$
\begin{aligned}
& =\frac{1}{m}\left\{\sum_{j=0}^{m-1}\left[\frac{(m-j) \lambda^{j}}{j !}\right]\left[\int_{t_{p}=0}^{\infty} t_{p}^{j} f_{p}\left(t_{p}\right) e^{-\lambda t_{p}} d t_{p}\right]\right\} \\
& =\frac{1}{m}\left\{\left.\sum_{j=0}^{m-1}\left[\frac{(m-j)(-\lambda)^{j}}{j !}\right]\left[\frac{d^{j} f_{p}^{*}(s)}{d s^{j}}\right]\right|_{s=\lambda}\right\}
\end{aligned}
$$

Consider a UMTS network with $n^{\prime}$-layer URA and $n^{\prime \prime}$-layer RA structure. From (9), (10) and (12), the probability mass function for $N_{u}$ is

$$
\operatorname{Pr}_{\left(n^{\prime}, n^{\prime \prime}\right)}\left[N_{u}=j\right]= \begin{cases}\operatorname{Pr}\left[N_{c}=j\right], & \text { for } j<K_{1} \\ \sum_{k=j}^{\infty} \operatorname{Pr}\left[N_{c}=k\right] P\left(n^{\prime}, k-K_{1}, j-K_{1}\right), & \text { for } K_{1} \leq j<K_{1}+K_{2} \\ \sum_{k=j}^{\infty} \sum_{i=K_{2}}^{k-K_{1}} \operatorname{Pr}\left[N_{c}=k\right] P\left(n^{\prime}, k-K_{1}, i\right) & \\ \times P\left(n^{\prime \prime}, i-K_{2}, j-K_{1}-K_{2}\right), & \text { for } j \geq K_{1}+K_{2}\end{cases}
$$

Equation (13) is explained as follows.

Case I: $j<K_{1}$. In this case, the MS only performs cell updates in the idle period. Therefore, the probability of $N_{u}$ location updates is equal to the probability of $N_{c}$ cell crossings.

Case II: $K_{1} \leq j<K_{1}+K_{2}$. In this case, there are $k$ cell crossings in the idle period (with probability $\operatorname{Pr}\left[N_{c}=k\right]$ ). Note that $k \geq j \geq K_{1}$. Therefore during these $k$ cell crossings, the MS performs $K_{1}$ cell updates for the first $K_{1}$ cell crossings (with probability 1). After the MS enters the URA update mode, the MS performs $j-K_{1}$ URA updates during the subsequent $k-K_{1}$ cell crossings (with probability $P\left(n^{\prime}, k-K_{1}, j-K_{1}\right)$ ).

Case III: $j \geq K_{1}+K_{2}$. Similar to Case II, $k$ represents the number of cell crossings in the idle period, where $k \geq j \geq K_{1}+K_{2}$. The MS first performs $K_{1}$ cell updates for the first $K_{1}$ cell crossings. Then it enters the URA update mode, where there are $i$ URA crossings in the remaining $k-K_{1}$ cell crossings with probability $P\left(n^{\prime}, k-K_{1}, i\right)$ (where $i \leq k-K_{1}$ ). During these $i$ URA crossings, the MS performs $K_{2}$ URA updates for the first $K_{2}$ URA crossings (with probability 1 ). Then the MS performs $j-K_{1}-K_{2}$ RA updates in the subsequent $i-K_{2}$ URA crossings with probability $P\left(n^{\prime \prime}, i-K_{2}, j-K_{1}-K_{2}\right)$ (where $\left.i \geq K_{2}\right)$.

Based on (13), we derive the net cost $C_{T}$ of location update and paging during the expected period $E\left[t_{p}\right]$. Assume that the cost for performing a location update is $U$ and the cost for paging at one 
cell is $V$. Let $C_{u}$ be the expected location update cost during $t_{p}$. From (13), we have

$$
C_{u}=U \sum_{j=0}^{\infty} j \operatorname{Pr}_{\left(n^{\prime}, n^{\prime \prime}\right)}\left[N_{u}=j\right]
$$

For the paging operation, there are three possibilities.

- If the MS stays at the cell update mode, then only one cell needs to page the MS.

- If the MS stays at the URA update mode, then all cells of the URA should page the MS.

- If the MS stays at the RA update mode, then all cells of the RA should page the MS.

Let $C_{v}$ be the expected paging cost during $t_{p}$. Based on (13), we have

$$
\begin{aligned}
C_{v}= & V\left\{\sum_{j=0}^{K_{1}-1} \operatorname{Pr}_{\left(n^{\prime}, n^{\prime \prime}\right)}\left[N_{u}=j\right]+S\left(n^{\prime}\right) \sum_{j=K_{1}}^{K_{1}+K_{2}-1} \operatorname{Pr}_{\left(n^{\prime}, n^{\prime \prime}\right)}\left[N_{u}=j\right]\right. \\
& \left.+S\left(n^{\prime}\right) S\left(n^{\prime \prime}\right) \sum_{j=K_{1}+K_{2}}^{\infty} \operatorname{Pr}_{\left(n^{\prime}, n^{\prime \prime}\right)}\left[N_{u}=j\right]\right\}
\end{aligned}
$$

From (14) and (15), the net cost $C_{T}$ for location update and paging during $t_{p}$ is

$$
C_{T}=C_{u}+C_{v}
$$

The analytical analysis has been validated by a discrete event simulation model. The simulation simulates the movement of an MS on the hexagonal plane where the UMTS network consists of 2-layer URAs and 2-layer RAs. The $t_{p}$ interval is exponentially distributed, and the cell residence times have the Erlang distribution given in (11). Note that our models can be applied to general $t_{p}$ distributions, and we only show the exponential case for demonstration. Table 2 compares the analytical and simulation results. The parameters $\lambda_{p 1}, \lambda_{p 2}$ and $\alpha$ used in Table 2 will be explained in detail later. The table indicates that the errors between the analytical and simulation models are within $1 \%$. Also, the errors for the $\theta_{1}(n, k), \theta_{2}(n, k), P\left(n, N_{m}, k\right)$ and $P^{*}\left(n, N_{m}^{*}, k^{*}\right)$ values are less than $1 \%$ in most cases. Details of these results will not be presented. It is clear that the analytical analysis is consistent with the simulation results. 
Table 2: Comparison between the analytical and simulation results

\begin{tabular}{|c|c|c|c|c|c|c|}
\hline \multicolumn{6}{|c|}{ Erlang $\lambda_{m}=\lambda, V_{m}=1 / \lambda^{2}\left(K_{2}=5, \lambda=\lambda_{p 1} / 30, \lambda_{p 2}=\lambda_{p 1} / 720, \alpha=0.6, U=4, V=1\right)$} \\
\hline$K_{1}$ & 1 & 5 & 9 & 13 & 17 & 21 \\
\hline$C_{T}$ (Analytical) & 25.2514 & 27.2976 & 29.1329 & 30.6918 & 32.0157 & 33.1403 \\
\hline$C_{T}$ (Simulation) $)$ & 25.5038 & 27.5381 & 29.3305 & 30.7652 & 32.1216 & 33.2821 \\
\hline Error & $0.9995 \%$ & $0.8810 \%$ & $0.6783 \%$ & $0.2392 \%$ & $0.3308 \%$ & $0.4279 \%$ \\
\hline \hline Erlang $\lambda_{m}=\lambda / 5, V_{m}=5 / \lambda^{2}\left(K_{1}=5, \lambda=\lambda_{p 1} / 30, \lambda_{p 2}=\lambda_{p 1} / 720, \alpha=0.6, U=4, V=1\right)$ \\
\hline$K_{2}$ & 0 & 4 & 8 & 12 & 16 & 20 \\
\hline$C_{T}($ Analytical $)$ & 13.9573 & 9.14372 & 8.26156 & 8.09384 & 8.06196 & 8.05589 \\
\hline$C_{T}$ (Simulation) $)$ & 14.0157 & 9.12147 & 8.23973 & 8.05265 & 8.03802 & 8.03023 \\
\hline Error & $0.4184 \%$ & $0.2439 \%$ & $0.2649 \%$ & $0.5115 \%$ & $0.2978 \%$ & $0.3195 \%$ \\
\hline \hline Erlang $\lambda_{m}=\lambda / 10, V_{m}=10 / \lambda^{2}\left(K_{2}=5, \lambda=\lambda_{p 1} / 30, \lambda_{p 2}=\lambda_{p 1} / 720, \alpha=0.6, U=4, V=1\right)$ \\
\hline$K_{1}$ & 1 & 5 & 9 & 13 & 17 & 21 \\
\hline$C_{T}($ Analytical $)$ & 5.61760 & 4.99601 & 4.87692 & 4.85365 & 4.84910 & 4.84822 \\
\hline$C_{T}$ (Simulation) & 5.58651 & 4.99591 & 4.88063 & 4.84336 & 4.84785 & 4.85471 \\
\hline Error & $0.5565 \%$ & $0.0020 \%$ & $0.0761 \%$ & $0.2125 \%$ & $0.0258 \%$ & $0.1339 \%$ \\
\hline
\end{tabular}

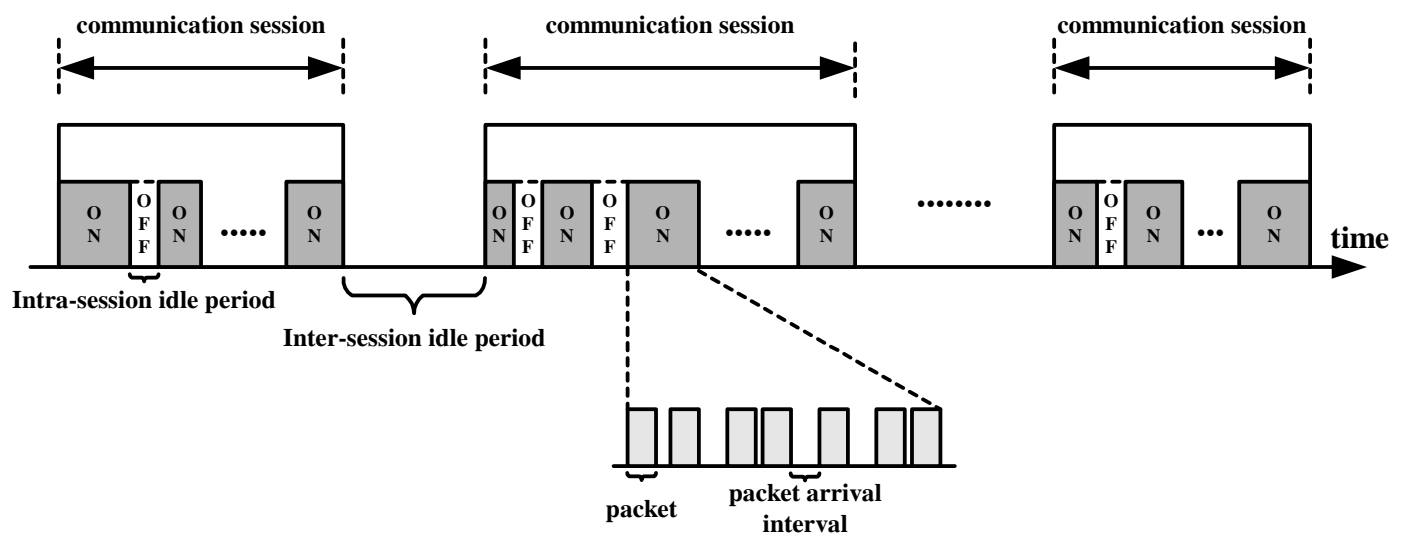

Figure 7: Packet data traffic 


\section{Numerical Examples}

Based on the simulation validated by the analytical model described in the previous section, this section investigates the performance of the UMTS inactivity counter mechanism. The experiment settings in this study are similar to that of our previous study on the GPRS Ready Counter mechanism [12]. We combine the ETSI packet data model [6] with the ON/OFF source model (also known as a packet train model) [4]. As shown in Figure 7, we assume that the packet data traffic consists of communication sessions. Within a communication session, packet traffic is characterized by $\mathrm{ON} / \mathrm{OFF}$ periods. In an ON-period, a burst of data packets are transmitted. In an OFF-period, no packets are delivered. Other assumptions are summarized as follows.

- The OFF-period $t_{p 1}$ is drawn from a Pareto distribution with mean $1 / \lambda_{p 1}$ and infinite variance. It has been shown that the Pareto distribution with infinite variance can match very well with the actual data traffic measurements [17]. A Pareto distribution has two parameters $\beta$ and $l$, where $\beta$ describes the "heaviness" of the tail of the distribution. The probability density function is $f_{p 1}\left(t_{p 1}\right)=\left(\frac{\beta}{l}\right)\left(\frac{l}{t_{p 1}}\right)^{\beta+1}$ and the expected value is $E\left[t_{p 1}\right]=\left(\frac{\beta}{\beta-1}\right) l$. If $\beta$ is between 1 and 2 , the variance for the distribution becomes infinite. The typical parameter values obtained in [17] are $E\left[t_{p 1}\right]=10.5$ seconds and $\beta=1.2$ for OFF-periods. Our study uses the above $E\left[t_{p 1}\right]$ and $\beta$ values.

- The idle period $t_{p 2}$ between two consecutive communication sessions has a Gamma distribution with mean $1 / \lambda_{p 2}$ and variance $V_{p 2}$. The Gamma distribution with shape parameter $\eta$ and scale parameter $\lambda$ (i.e., mean $1 / \lambda_{p 2}=\eta / \lambda$ and variance $V_{p 2}=\eta / \lambda^{2}$ ) has the following density function

$$
f_{p 2}(t)=\frac{\lambda e^{-\lambda t}(\lambda t)^{\eta-1}}{\Gamma(\eta)} \quad \text { for } t \geq 0
$$

where $\Gamma(\eta)=\int_{z=0}^{\infty} z^{\eta-1} e^{-z} d z$ is the Gamma function. It has been shown that the distribution of any positive random variable can be approximated by a mixture of Gamma distributions (see Lemma 3.9 in [11]). In this paper we use the Gamma distribution to investigate the impact of variance for inter-session idle periods.

- Following the ETSI packet data model, the number of OFF-periods in a session has a geometric distribution with mean $\alpha /(1-\alpha)$, where $0 \leq \alpha<1$. In other words, an ON-period 


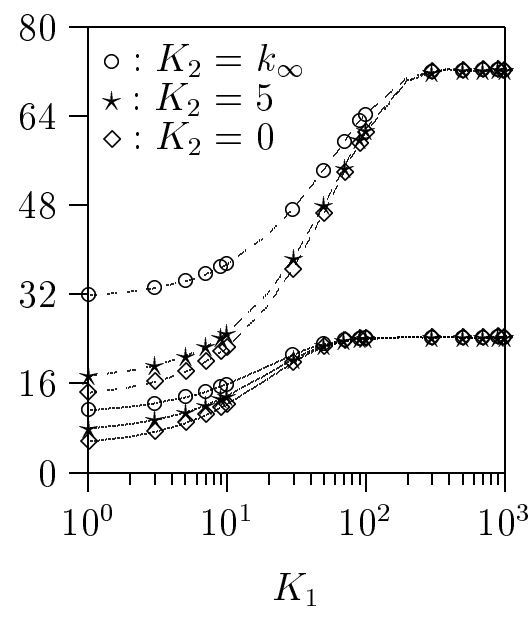

(a) The $C_{u}$ cost

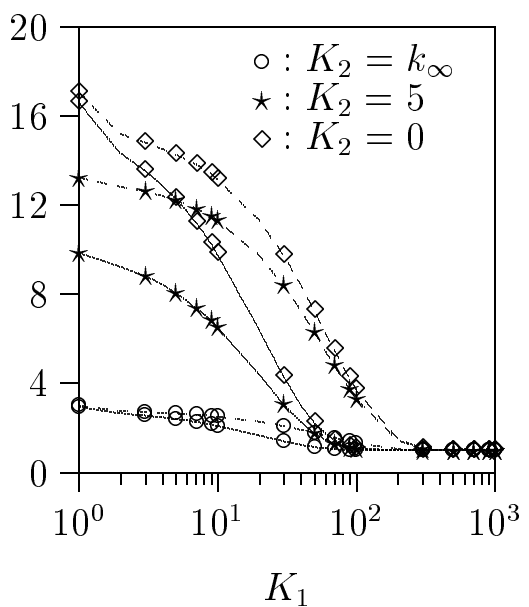

(b) The $C_{v}$ cost

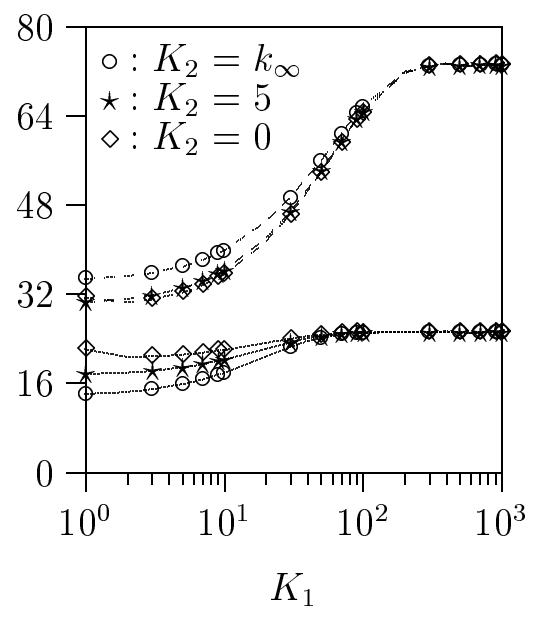

(c) The $C_{T}$ cost

Figure 8: Effects of $K_{1}, K_{2}$ and $\lambda_{p 2}$ on $C_{u}, C_{v}$ and $C_{T}$ (solid: $\lambda_{p 2}=\frac{1}{200} \lambda_{p 1}$; dashed: $\lambda_{p 2}=\frac{1}{600} \lambda_{p 1}$; $\lambda_{m}=\frac{1}{10} \lambda_{p 1}, V_{m}=\frac{1}{\lambda_{m}^{2}}, V_{p 2}=\frac{1}{\lambda_{p 2}^{2}}, \frac{U}{V}=4, \alpha=0.7$ )

Table 3: The $C_{u}$ and $C_{v}$ costs in a $t_{p 2}$ period $\left(\lambda_{p 1}=10 \lambda_{m}\right.$; the costs are normalized by one LU cost)

\begin{tabular}{|c|c|c|}
\hline$K_{1}$ and $K_{2}$ & $\lambda_{m}=20 \lambda_{p 2}$ & $\lambda_{m}=60 \lambda_{p 2}$ \\
\hline$K_{1}=k_{\infty}, K_{2}=k_{\text {any }}$ & $C_{T}=20.25$ & $C_{T}=60.25$ \\
\hline$K_{1}=1, K_{2}=k_{\infty}$ & $C_{T}=10.8$ & $C_{T}=28.0$ \\
\hline$K_{1}=1, K_{2}=0$ & $C_{T}=16.1$ & $C_{T}=23.9$ \\
\hline
\end{tabular}

is followed by an OFF-period with probability $\alpha$, and is followed by an inter-session idle period with probability $1-\alpha$.

- The cell residence times have a Gamma distribution with the mean $1 / \lambda_{m}$ and the variance $V_{m}$. The Gamma distribution was employed to model MS movement in many studies $[5,8$, 9], and is used in this paper to investigate the impact of variance for cell residence times.

To simplify our discussion, we consider the 2-layer URA and 2-layer RA cell layout. The effects of the input parameters are investigated as follows.

Effects of $K_{1}$. Figure 8 shows how $K_{1}$ affects $C_{u}, C_{v}$ and $C_{T}$. For a fixed $K_{2}$, it is clear that if $K_{1}$ increases, the location update (LU) $\operatorname{cost} C_{u}$ increases while the paging cost $C_{v}$ decreases. 
When $K_{1}$ is very large, the MS always performs cell updates, and no URA or RA update is executed. In this case, the $C_{u}$ and $C_{v}$ costs are not affected by the change of $K_{1}$ (see the $C_{u}$ and $C_{v}$ curves in Figures 8(a) and (b) where $K_{1} \geq 10^{2}$ ). In Figure 8(c), the $C_{T}$ is computed directly from the $C_{u}$ and $C_{v}$ using Equation (16), where $\frac{U}{V}=4$. For the input parameters selected in Figure 8(c), the lowest $C_{T}$ costs are observed when $K_{1}=1$ or 2 . The selection of $K_{1}$ is affected by $t_{p 1}$ and $t_{p 2}$, which are described in the following two cases.

Case I. Since the OFF-periods $t_{p 1}$ are short (specifically, $E\left[t_{p 1}\right]=\frac{1}{10 \lambda_{m}}$ in Figure 8), there are few cell updates during $t_{p 1}$ and the paging cost $C_{v}$ dominates the $C_{T}$ cost. Therefore, to maintain low $C_{v}$ cost (i.e., to ensure that only one cell is paged for the next packet delivery), the MS should stay at the cell update mode. For all experiments considered in this paper, the probability that there are less than 2 cell crossings during $t_{p 1}$ is larger than $99 \%$. Therefore if $K_{1} \geq 2$ is selected, then it is likely that the MS will stay at the cell update mode in the $t_{p 1}$ period.

Case II. On the other hand, the MS crosses many cell boundaries during an inter-session idle period $t_{p 2}$. For example, the average number of cell crossings is 20 for $\lambda_{m}=20 \lambda_{p 2}$ and 60 for $\lambda_{m}=60 \lambda_{p 2}$. Consider the case where $\lambda_{m}=20 \lambda_{p 2}$. Table 3 lists $C_{u}$ and $C_{v}$ normalized by one location update (LU) cost. When $K_{1}=k_{\infty}{ }^{1}$ and $K_{2}=k_{\text {any }}{ }^{2}$, the $C_{T}$ cost is 20.25 LUs. When $K_{1}=1$ and $K_{2}=k_{\infty}$, the $C_{T}$ cost is 10.8 LUs. Based on the above discussion, we have

$$
C_{T}\left(K_{1}=1\right)<C_{T}\left(K_{1}=k_{\infty}\right)
$$

Since Theorem 1 in Appendix A indicates that the lowest $C_{T}$ value occurs when $K_{1}=1$ or $K_{1}=k_{\infty},(17)$ implies that $K_{1}=1$ is the optimal threshold value in a $t_{p 2}$ period.

To obtain low $C_{T}$ values by considering both Cases I and II, it is appropriate to select $K_{1}=1$ or 2 .

Effects of $K_{2}$. Figures 8 and 9 show how $K_{2}$ affects $C_{u}, C_{v}$ and $C_{T}$. The effects of $K_{2}$ on the location update cost $C_{u}$ and paging cost $C_{v}$ are similar to that of $K_{1}$ : given a $K_{1}$ value, the $C_{u}$ cost is an increasing function of $K_{2}$ while the $C_{v}$ cost is a decreasing function of $K_{2}$.

\footnotetext{
${ }^{1}$ In computer simulation, it is impossible to generate an infinite number. Thus, $k_{\infty}$ is defined as a number larger than the number of cell crossings in any idle period.

${ }^{2} k_{a n y}$ is an arbitrary integer number.
} 


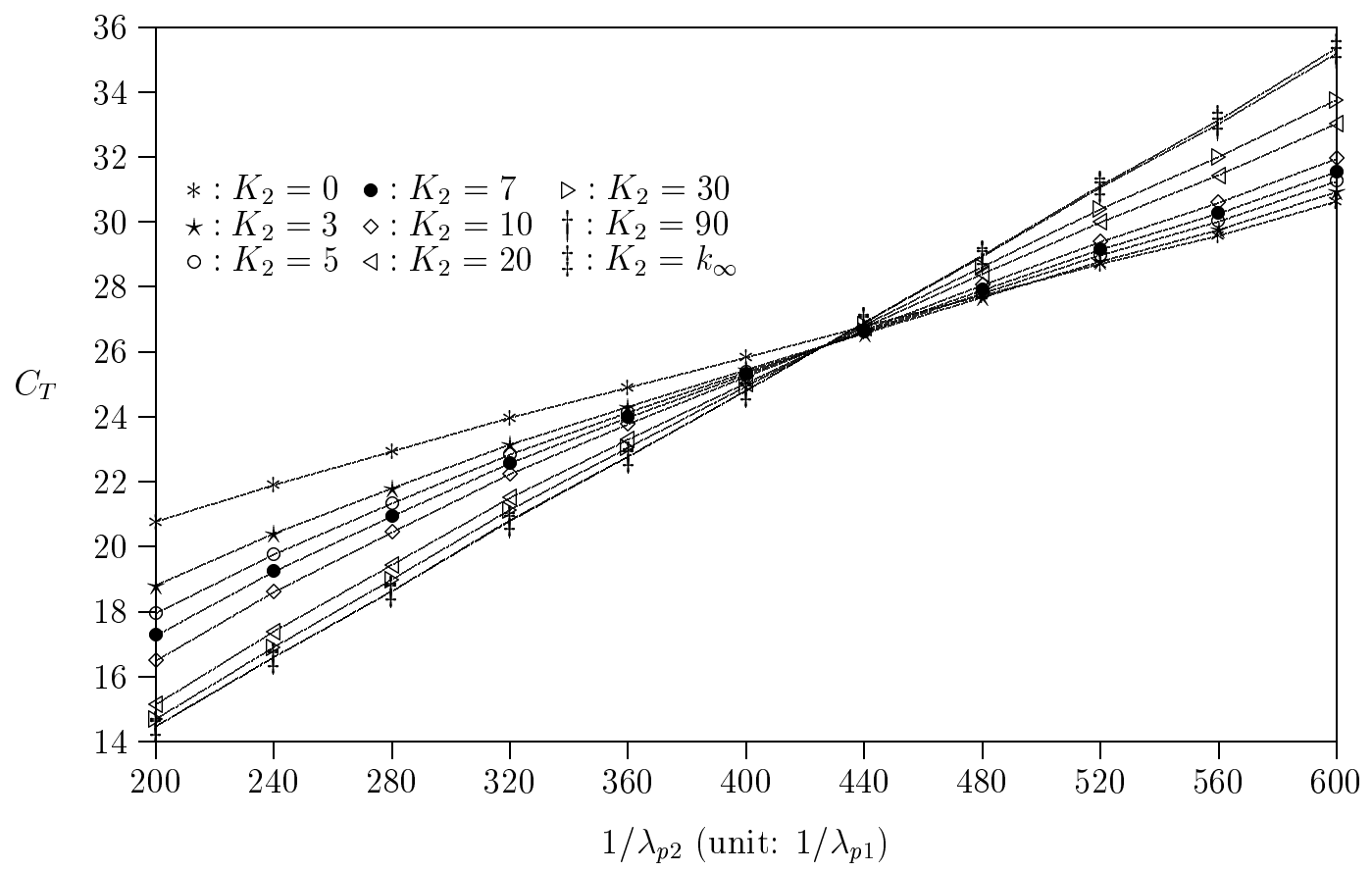

Figure 9: Effects of $K_{2}$ on $C_{T}\left(K_{1}=2, \lambda_{m}=\frac{1}{10} \lambda_{p 1}, V_{m}=\frac{1}{\lambda_{m}^{2}}, V_{p 2}=\frac{1}{\lambda_{p 2}^{2}}, \frac{U}{V}=4, \alpha=0.7\right)$

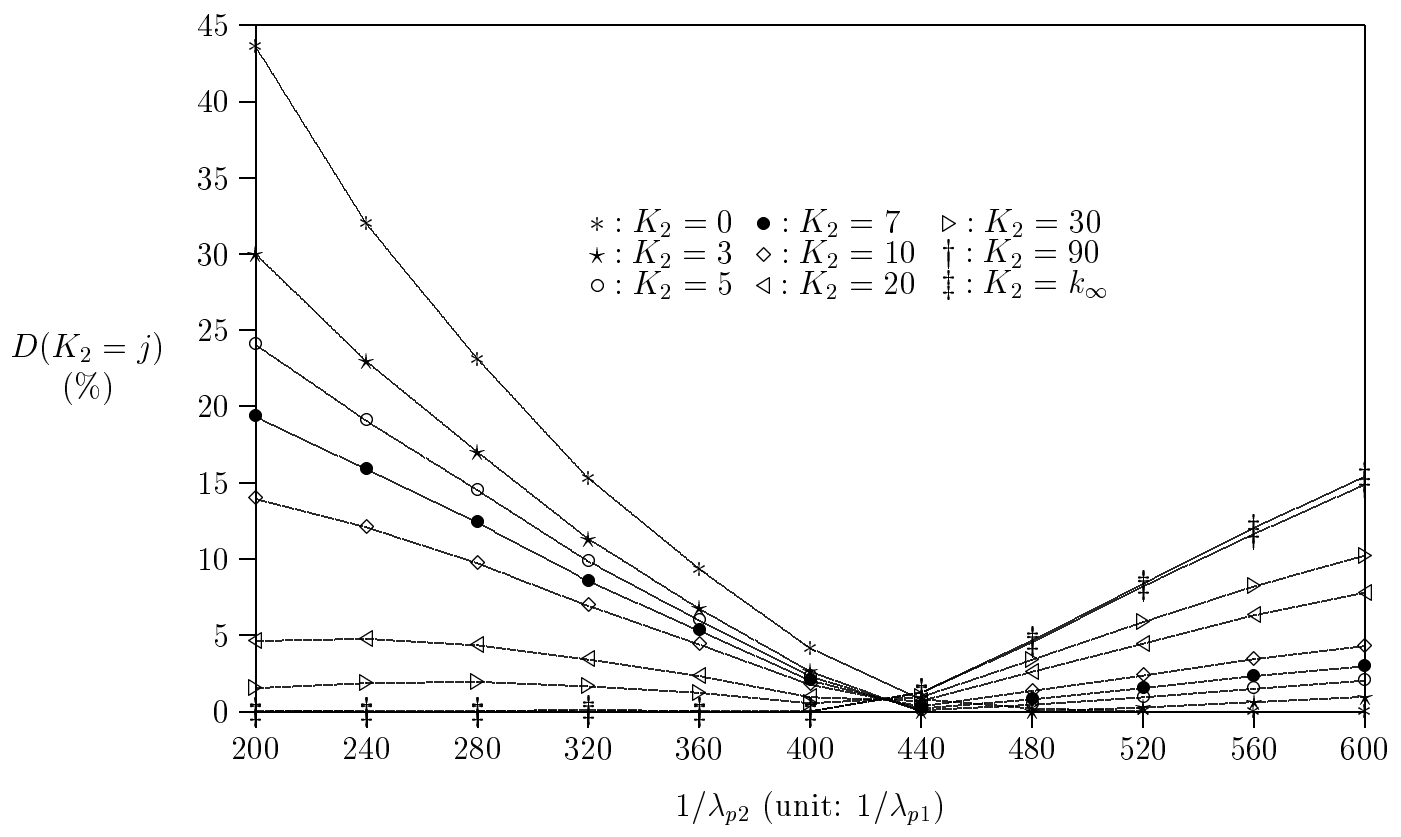

Figure 10: The discrepancy $D\left(K_{2}=j\right)$ of $C_{T}\left(K_{1}=2, \lambda_{m}=\frac{1}{10} \lambda_{p 1}, V_{m}=\frac{1}{\lambda_{m}^{2}}, V_{p 2}=\frac{1}{\lambda_{p 2}^{2}}, \frac{U}{V}=4\right.$, $\alpha=0.7$ ) 
Figure 8 also indicates that when $K_{1}$ is large, $C_{u}$ and $C_{v}$ are not affected by the change of $K_{2}$. Figure 9 shows that if $\lambda_{p 2}$ is small, $C_{T}$ is an increasing function of $K_{2}$. On the other hand, if $\lambda_{p 2}$ is large, $C_{T}$ is a decreasing function of $K_{2}$. This phenomenon is explained in the following two cases.

Case I. $\lambda_{p 2}=\frac{1}{600} \lambda_{p 1}$ (the $t_{p 2}$ period is long). When $K_{1}=1$ and $K_{2}=k_{\infty}$, Table 3 indicates that the $C_{T}$ cost is 28 LUs. When $K_{1}=1$ and $K_{2}=0$, the $C_{T}$ cost is 23.9 LUs. Thus,

$$
C_{T}\left(K_{2}=0\right)<C_{T}\left(K_{2}=k_{\infty}\right)
$$

From Theorem 1, the lowest $C_{T}$ value occurs when $K_{2}=0$ or $K_{2}=k_{\infty}$, and (18) implies that the lowest $C_{T}$ cost is expected when $K_{2}=0$.

Case II. $\lambda_{p 2}=\frac{1}{200} \lambda_{p 1}$ (the $t_{p 2}$ period is short). The $C_{T}$ cost is 10.8 LUs when $K_{1}=1$ and $K_{2}=k_{\infty}$, and is 16.1 LUs when $K_{1}=1$ and $K_{2}=0$. In this case, Theorem 1 indicates that the lowest $C_{T}$ cost is observed when $K_{2}=k_{\infty}$.

In a real mobile communications network, the mobility and traffic patterns of a mobile user usually change dynamically. To obtain lower $C_{T}$ cost in such an environment, it is required to consider both high (i.e., large $\lambda_{p 2}$ ) and low (i.e., small $\lambda_{p 2}$ ) packet arrival patterns. Figure 10 illustrates the discrepancies $D\left(K_{2}=j\right)$ of $C_{T}$ for various $\lambda_{p 2}$ values, when $K_{2}=j$. The discrepancy $D\left(K_{2}=j\right)$ is defined as

$$
D\left(K_{2}=j\right)=\frac{C_{T}\left(K_{2}=j\right)-C_{T}^{*}}{C_{T}^{*}}
$$

where $C_{T}\left(K_{2}=j\right)$ is the $C_{T}$ cost for $K_{2}=j$ and $C_{T}^{*}$ is the lowest $C_{T}$ cost. Under a given $\lambda_{p 2}$ value, Figure 9 indicates that there exists an optimal value $K_{2}^{*}$ that results in the lowest $C_{T}^{*}$. Figure 10 plots the $D\left(K_{2}=j\right)$ curves for various $\lambda_{p 2}$. When $\lambda_{p 2}=\frac{1}{200} \lambda_{p 1}$, Figure 9 shows that the optimal $K_{2}^{*}$ is $k_{\infty}$ and the corresponding $C_{T}^{*}$ is 14.46 . Since $C_{T}\left(K_{2}=0\right)$ is 20.76 , the discrepancy $D\left(K_{2}=0\right)$ is $((20.76-14.46) / 14.46) * 100 \simeq 43.57 \%$.

Figure 10 indicates that when $\lambda_{p 2}<\frac{1}{440} \lambda_{p 1}, C_{T}$ is an increasing function of $K_{2}$. In this case, the discrepancies $D\left(K_{2}=j\right)$ for $j<30$ are less than $10 \%$. On the other hand, when $\lambda_{p 2}>\frac{1}{440} \lambda_{p 1}, C_{T}$ is a decreasing function of $K_{2}$. In this case, $D\left(K_{2}=j\right)$ are less than $10 \%$ for $j>20$. Therefore, for all $\lambda_{p 2}$ values considered in our experiments, if we want to maintain reasonable low $C_{T}$ values such that $D\left(K_{2}=j\right)<10 \%$, the $K_{2}$ value should be selected from the range $[20,30]$. 


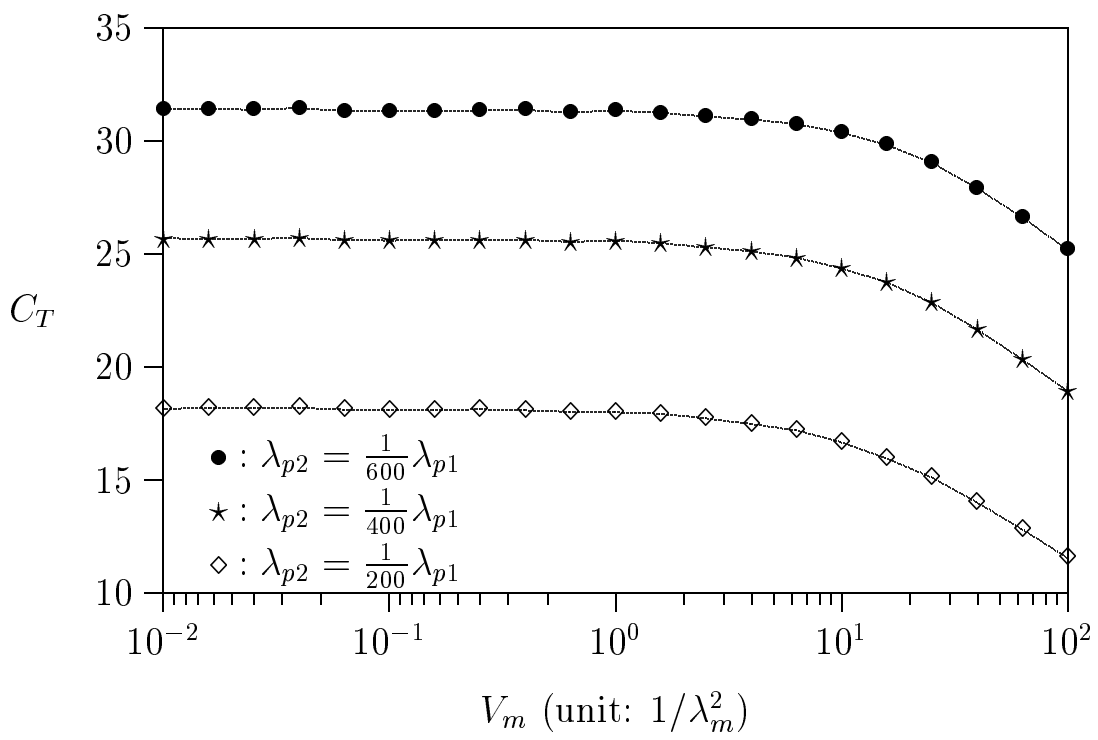

Figure 11: Effects of $V_{m}$ on $C_{T}\left(K_{1}=2, K_{2}=5, \lambda_{m}=\frac{1}{10} \lambda_{p 1}, V_{p 2}=\frac{1}{\lambda_{p 2}^{2}}, \frac{U}{V}=4, \alpha=0.7\right)$

Effects of $\lambda_{p 2}$. Figures 8(a), (b) and (c) plot the $C_{u}, C_{v}$ and $C_{T}$ curves for $\lambda_{p 2}=\frac{1}{200} \lambda_{p 1}$ and $\lambda_{p 2}=\frac{1}{600} \lambda_{p 1}$. The figures show that $C_{u}, C_{v}$ and $C_{T}$ increase as $\lambda_{p 2}$ decreases. A small $\lambda_{p 2}$ implies a long inter-session idle period and more cell movements during this period. Therefore, the location update $\operatorname{cost} C_{u}$ will increase accordingly. For fixed $K_{1}$ and $K_{2}$ values, increasing the number of cell movements implies increasing the probability that the MS will enter the URA update mode or even the RA update mode when the next packet arrives. Thus, high paging cost is expected. We also notice that when both $K_{1}$ and $\lambda_{p 2}$ are large, increasing $K_{1}$ only has insignificant effect on $C_{T}$. This phenomenon is explained as follows. For large $\lambda_{p 2}$ and $K_{1}$, it is likely that $K_{1}$ is larger than the number of cell crossings during the idle period. Therefore, the MS will only perform cell updates, and increasing $K_{1}$ only insignificantly increases the $C_{T}$ value.

Effects of $\alpha$. A smaller $\alpha$ implies more inter-session idle periods. Since more cell crossings are observed in an inter-session idle period than in the OFF-periods of a session, $C_{T}$ increases as $\alpha$ decreases. Similar to the discussion for the interaction between $K_{1}$ and $\lambda_{p 2}, C_{T}$ is more sensitive to the change of $K_{1}$ for a small $\alpha$ than a large $\alpha$.

Effects of variance $V_{m}$. Figure 11 plots the $C_{T}$ curves where the cell residence times have a Gamma distribution with the mean $1 / \lambda_{m}$ and the variance $V_{m}$. This figure indicates that 


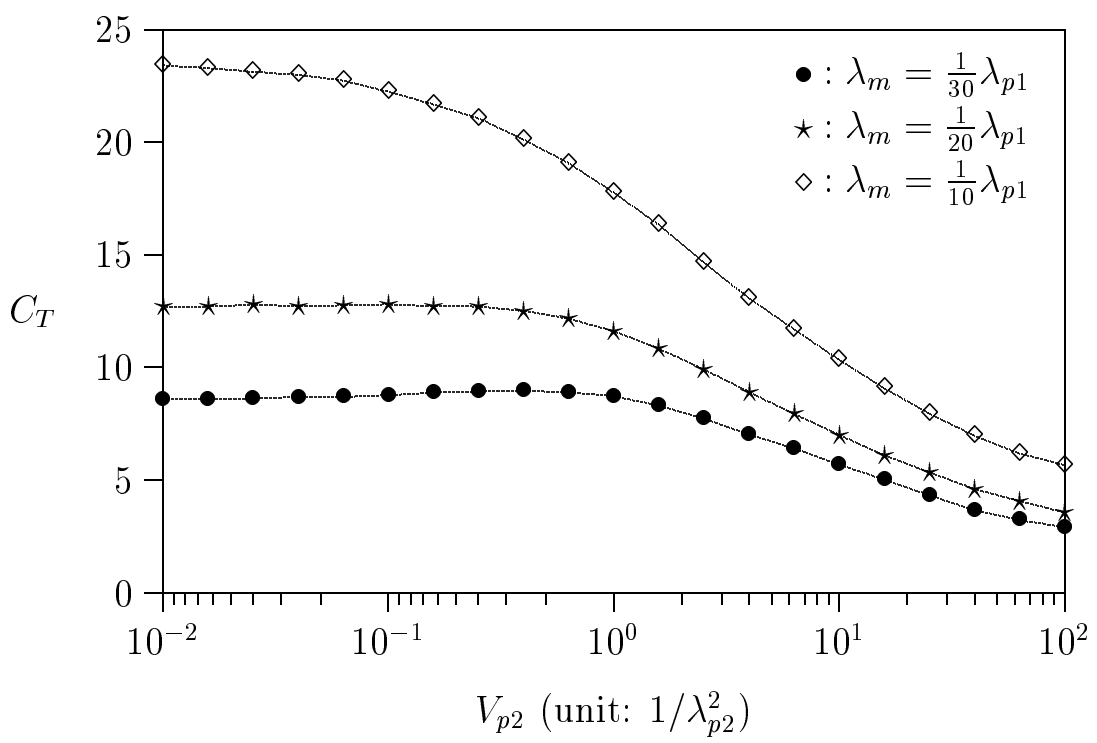

Figure 12: Effects of $V_{p 2}$ on $C_{T}\left(K_{1}=2, K_{2}=5, \lambda_{p 2}=\frac{1}{200} \lambda_{p 1}, V_{m}=\frac{1}{\lambda_{m}^{2}}, \frac{U}{V}=4, \alpha=0.7\right)$

$C_{T}$ is a decreasing function of $V_{m}$. When $V_{m}$ increases, more short and long cell residence times are observed. Long cell residence times imply small number of cell crossings $N_{c}$, which result in a small $C_{T}$. On the contrary, short cell residence times imply large number of cell crossings $N_{c}$, which increases $C_{T}$. We observe that, when $N_{c}>K_{1}$, the numbers of URA crossings and RA crossings do not increase as fast as $N_{c}$ does. The result is that the negative effect of short cell residence times are not as significant as the positive effect of long cell residence times. Therefore, the combined effect is that $C_{T}$ decreases as $V_{m}$ increases. Figure 11 also indicates that $C_{T}$ is not sensitive to the change of $V_{m}$ when $V_{m} \leq \frac{1}{\lambda_{m}^{2}}$.

Effects of variance $V_{p 2}$. Figure 12 plots $C_{T}$ as a function of $V_{p 2}$, where $V_{p 2}$ is the variance of the inter-session idle periods $t_{p 2}$. This figure shows that $C_{T}$ decreases as $V_{p 2}$ increases. When $V_{p 2}$ increases, more long and short $t_{p 2}$ periods are observed. For a short $t_{p 2}$, few cell movements occur during this period (i.e., $N_{c}$ is small) and a small $C_{T}$ is expected. On the other hand, for a long $t_{p 2}$, many cell movements occur during this period (i.e., $N_{c}$ is large) and a large $C_{T}$ is expected. Nevertheless, when $N_{c}>K_{1}$, the numbers of URA crossings and RA crossings do not increase as rapidly as $N_{c}$ does. Consequently, the net effect is that $C_{T}$ decreases as $V_{p 2}$ increases. 


\section{Conclusions}

This paper investigated the location management strategy for UMTS PS service domain. When an MS is not in any communication session, the system tracks the RA where the MS resides. Within a communication session, the MS is tracked at the cell level during packet transmission. In the idle period of an ongoing session, the MS is tracked at the URA level to avoid frequent cell updates while still keeping the radio connection. The inactivity counter mechanism was proposed in $3 \mathrm{GPP}$ 25.331 [1] to determine when to switch between the three location tracking modes (cell, URA or RA). In this mechanism, two inactivity counters are used to count the numbers of cell updates and URA updates in an idle period between two packet transmissions. If the number of cell updates reaches a threshold $K_{1}$, the MS is switched from the cell tracking to the URA tracking. After that, if the number of URA updates reaches a threshold $K_{2}$, the MS is tracked at the RA level. We utilized analytical and simulation models to investigate the performance of the inactivity counter mechanism. It is clear that as $K_{1}$ and $K_{2}$ increase, the location update cost increases while the paging cost decreases. There exists optimal $K_{1}$ and $K_{2}$ that minimize the net cost $C_{T}$ of location update and paging. For the input parameters considered in this paper, the lowest $C_{T}$ costs are observed when $K_{1}=1$ or 2 . If the inter-session idle periods $t_{p 2}$ are long, $C_{T}$ is an increasing function of $K_{2}$. On the other hand, if the $t_{p 2}$ are short, $C_{T}$ is a decreasing function of $K_{2}$. We quantitatively showed how $C_{T}$ increases as inter-session idle periods and user mobility increase. In addition, both the variances $V_{m}$ of cell residence times and $V_{p 2}$ of inter-session idle periods affect $C_{T}$. Our study indicated that as $V_{m}$ and $V_{p 2}$ increase, $C_{T}$ decreases.

\section{Acknowledgments}

The authors would like to thank the anonymous reviewers. Their comments have significantly improved the quality of this paper.

\section{A Proof for Theorem 1}

Consider an idle period where no packet is delivered. Let $N_{c}$ be the number of cell crossings in this

idle period. In the inactivity counter algorithm, let $C_{T}\left(K_{1}, K_{2}\right)$ be the net cost of location update 
and paging in the idle period with cell update threshold $K_{1}$ and URA update threshold $K_{2}$. Let $K^{*}=\left(K_{1}^{*}, K_{2}^{*}\right)$ be the optimal threshold value pair that minimizes the net cost $C_{T}$. Define $k_{\infty}$ as a number larger than the $N_{c}$ value in the idle period. Let $k_{a n y}$ be an arbitrary integer number.

Theorem 1. For an idle period, $K^{*}=\left(k_{\infty}, k_{\text {any }}\right),\left(1, k_{\infty}\right)$ or $(1,0)$.

Proof. Let $N_{u r}(j)$ and $N_{r}(j)$ be the numbers of URA crossings and RA crossings between the $j+1$-st cell crossing and the $N_{c}$-th cell crossing, respectively. It is clear that $j<N_{c}$. We consider three cases.

Case I: $N_{c}<K_{1}$. In this case, there are no URA and RA updates during the idle period, and the net cost $C_{T}$ is not affected by the value of $K_{2}$. Therefore, for an arbitrary $K_{2}$ value $k_{a n y}$, we have

$$
C_{T}\left(N_{c}+1, k_{\text {any }}\right)=C_{T}\left(N_{c}+2, k_{\text {any }}\right)=\cdots=C_{T}\left(k_{\infty}, k_{\text {any }}\right)
$$

Case II: $N_{c} \geq K_{1}$ and $N_{u r}\left(K_{1}\right)<K_{2}$. In this case, there are no RA updates during the idle period. The net cost $C_{T}$ is

$$
C_{T}\left(K_{1}, K_{2}\right)=U\left[K_{1}+N_{u r}\left(K_{1}\right)\right]+S_{U R A} V
$$

where $S_{U R A}$ is the number of cells in an URA (e.g., $S(n)$ in Equation (5) for an $n$-layer URA layout). Note that the number of URA crossings between the 2-nd cell crossing and the $K_{1}$-th cell crossing is $N_{u r}(1)-N_{u r}\left(K_{1}\right)$. Since the number of URA crossings is no more than the number of cell crossings in the same time period, we have

$$
N_{u r}(1)-N_{u r}\left(K_{1}\right) \leq K_{1}-1 \text { or } K_{1}+N_{u r}\left(K_{1}\right) \geq 1+N_{u r}(1)
$$

From (20) and (21), we have

$$
\begin{aligned}
C_{T}\left(K_{1}, K_{2}\right) & \geq U\left[1+N_{u r}(1)\right]+S_{U R A} V \\
& =C_{T}\left(1, N_{u r}(1)+1\right)=C_{T}\left(1, N_{u r}(1)+2\right)=\cdots=C_{T}\left(1, k_{\infty}\right)
\end{aligned}
$$

Case III: $N_{c} \geq K_{1}$ and $N_{u r}\left(K_{1}\right) \geq K_{2}$. Let the $K_{2}$-th URA update correspond to the i-th cell crossing in this idle period, then $N_{r}(i)$ is the number of RA updates and the net cost is

$$
C_{T}\left(K_{1}, K_{2}\right)=U\left[K_{1}+K_{2}+N_{r}(i)\right]+S_{R A} V
$$


where $S_{R A}$ is the number of cells in an RA. The number of RA crossings between the 2-nd cell crossing and the $\mathrm{i}$-th cell crossing is $N_{r}(1)-N_{r}(i)$. Similar to the derivation of (21),

$$
N_{r}(1)-N_{r}(i) \leq K_{1}-1+K_{2} \text { or } K_{1}+K_{2}+N_{r}(i) \geq 1+N_{r}(1)
$$

From (23) and (24), we have

$$
C_{T}\left(K_{1}, K_{2}\right) \geq U\left[1+N_{r}(1)\right]+S_{R A} V=C_{T}(1,0)
$$

From (19), (22) and (25), for all $K_{1}$ and $K_{2}$ values we have

$$
C_{T}\left(K_{1}, K_{2}\right) \geq \min \left[C_{T}\left(k_{\infty}, k_{a n y}\right), C_{T}\left(1, k_{\infty}\right), C_{T}(1,0)\right]
$$

In other words, $K^{*}=\left(k_{\infty}, k_{\text {any }}\right),\left(1, k_{\infty}\right)$ or $(1,0)$.

\section{References}

[1] 3GPP. 3rd Generation Partnership Project; Technical Specification Group Radio Access Network; RRC Protocol Specification for Release 1999. Technical Specification 3G TS 25.331 version 3.5.0 (2000-12), 2000.

[2] 3GPP. 3rd Generation Partnership Project; Technical Specification Group Services and Systems Aspects; General Packet Radio Service (GPRS); Service Description; Stage 2. Technical Specification 3G TS 23.060 version 3.6.0 (2001-01), 2000.

[3] Akyildiz, I.F., Lin, Y.-B., Lai, W.-R., and Chen, R.-J. A New Random Walk Model for PCS Networks. IEEE Journal on Selected Areas in Communications, 18(7):1254-1260, July 2000.

[4] Cheng, M. and Chang, L.F. Wireless Dynamic Channel Assignment Performance Under Packet Data Traffic. IEEE Journal on Selected Areas in Communications, 17(7):1257-1269, July 1999.

[5] Chlamtac, I., Fang, Y., and Zeng, H. Call Blocking Analysis for PCS Networks under General Cell Residence Time. IEEE Wireless Communications and Networking Conference (WCNC), New Orleans, September 1999. 
[6] ETSI. UMTS Terrestrial Radio Access (UTRA); Concept Evaluation, Version 3.0.0. Technical Report UMTS 30.06, December 1997.

[7] Fang, Y. Hyper-Erlang Distribution Model and its Application in Wireless Mobile Networks. ACM-Baltzer J. Wireless Networks, 7(3):211-219, May 2001.

[8] Fang, Y. and Chlamtac, I. Teletraffic Analysis and Mobility Modeling for PCS Networks. IEEE Transactions on Communications, 47(7):1062-1072, July 1999.

[9] Fang, Y., Chlamtac, I., and Fei, H.-B. Analytical Results for Optimal Choice of Location Update Interval for Mobility Database Failure Restoration in PCS networks. IEEE Transactions on Parallel and Distributed Systems, 11(6):615-624, June 2000.

[10] Holma, H. and Toskala, A. WCDMA for UMTS. John Wiley \& Sons, Inc., 2000.

[11] Kelly, F.P. Reversibility and Stochastic Networks. John Wiley \& Sons, 1979.

[12] Lin, Y.-B. and Yang, S.-R. A Mobility Management Strategy for GPRS. IEEE Transactions on Wireless Communications, 2(6), November 2003.

[13] Lin, Y.-B., Lai, W.-R., and Chen, R.-J. Performance Analysis for Dual Band PCS Networks. IEEE Transactions on Computers, 49(2):148-159, 2000.

[14] Manber, U. Introduction to Algorithms - A Creative Approach. Addison Wesley., 1989.

[15] Nelson, R. Probability, Stochastic Processes, and Queueing Theory. Springer-Verlag., 1995.

[16] Tseng, Y.-C. and Hung, W.-N. An Improved Cell Type Classification for Random Walk Modeling in Cellular Networks. IEEE Communications Letters, 5(8):337-339, August 2001.

[17] Willinger, W., Taqqu, M.S., Sherman, R., and Wilson, D.V. Self-Similarity through HighVariability: Statistical Analysis of Ethernet LAN Traffic at the Source Level. IEEE/ACM Trans. Networking, 5(1):71-86, February 1997. 